Check for updates

Cite this: J. Mater. Chem. A, 2019, 7, 20444

DOI: $10.1039 /$ c9ta90200g

www.rsc.org/MaterialsA

\section{Retraction: Mesoporous amorphous FeOF nanococoons for high-rate and long-life rechargeable sodium-ion batteries}

\author{
Dong Ge Tong*
}

Retraction of 'Mesoporous amorphous FeOF nanococoons for high-rate and long-life rechargeable sodium-ion batteries' by Shi Yan Fu et al., J. Mater. Chem. A, 2015, 3, 16716-16727.

The Royal Society of Chemistry hereby wholly retracts this Journal of Materials Chemistry A article, with the agreement of the authors, due to concerns with the reliability of the electron microscope (EM) and XRD images in the published article.

The STEM images in Fig. 1a, S5a, S8, S9, S12, S13e, S14a and S26a contain repetitions of distinct shapes or patterns within the images.

Repeating patterns can be observed in the baselines of the XRD spectra in S27.

The authors informed us that the characterisation was completed by a third party company and they used the images "without any editing or modification". The authors repeated the experiments and requested to provide replacement data for Fig. 1a, S5a, S8, S9, S12, S13e, S14a, S26a and S27. The new figures were reviewed by an independent expert. The authors believe that the scientific content and conclusions of the related studies presented by the pictures in the published paper can be reproduced. However, the independent expert still questions the reliability of the published images. The authors informed us that due to a flooding accident in the laboratory, the original data of the published EM images were destroyed. In addition, the third party company only saved the test data for one month. Due to the large number of images, it is not possible to replace the published images with the new figures. To avoid the possibility of publishing unreliable EM images, the authors agree to retract this paper to protect the rigor of the scientific record.

This retraction supersedes the information provided in the Expression of Concern related to this article.

Signed: Dr Dong Ge Tong (on behalf of the authors)

Date: $1^{\text {st }}$ August 2019

Retraction endorsed by Sam Keltie, Executive Editor, Journal of Materials Chemistry A 